\title{
Stochastic epidemiological models: approximations and stability - overview
}

\begin{abstract}
In this short paper we give an overview of new stochastic epidemic and endemic models. Stochastic behavior is caused by random media in the form of coefficients of the models depending on semi-Markov process, which switches the states of the system under consideration. For both models we consider approximation principles and stability. The approximations can be considered in many forms, including averaging (resulting system is deterministic), merging (resulting system is stochastic but with Markov switching), and discussion approximation (resulting system is described in the form of the system of stochastic differential equations). Stochastic stability of vector stochastic differential equations and of averaged systems (specifically SARS model) is considered as well.
\end{abstract}

Keywords: stochastic epidemic SIR model, stochastic endemic SIR model, semimarkov random media, averaging principle, averaged endemic SIR model, stability of SARS model, two-state markov chain, stochastic stability of vector SDEs
Volume 2 Issue 7 - 2015

\author{
Anatoliy Swishchuk \\ Department of Mathematics and Statistics, University of Calgary, \\ Canada
}

\begin{abstract}
Correspondence: Anatoliy Swishchuk, Department of Mathematics and Statistics, University of Calgary, Calgary, Canada,Email aswish@ucalgary.ca
\end{abstract}

Received: September 02, 2015 | Published: October 27, 2015

\section{Overview on stochastic epidemic and endemic models}

In the last years, deterministic and stochastic epidemic models, in both discrete and continuous time, have been studied. ${ }^{1-3}$ Both model types are needed, and both have their advantages and weaknesses..$^{4-5}$ The deterministic models lead to power full qualitative results with important threshold behavior. ${ }^{6}$ They can serve as a useful inside into the stochastic models as well.

Some recent publications have shown the importance of stochastic epidemiological models, their approximations and stability.

Stochastic epidemiological models can be constructed in two ways: 1) as a system of stochastic differential equations (e.g.,marine bacteriophaque population), and 2) as a system of differential equation with coefficients depending on some stochastic process (e.g., semiMarkov process, in general case), switching the modes/states of the system under consideration (e.g., stochastic SIR models in random media). Stochastic stability of vector stochastic differential equations and its application to a stochastic epidemic model are considered in Swishchuk, Svishchuk and Limnios. ${ }^{8}$ Stochastic SARS model and its approximations and stability are considered in Swishchuk, Limnios and Svishchuk. ${ }^{8}$ Stochastic endemic SIR model in random media is considered in Svishchuk. ${ }^{9}$ Approximations of stochastic models (that can be complicated and have a complex structure) are based on general limit theory for vector differential equations with random coefficients. ${ }^{7}$ Stability of stochastic models is based on general stability theory for vector stochastic differential equations..$^{10}$ In the section below we give two specific examples of stochastic epidemiological models.

\section{Stochastic SARS models: in semi-markov random media and with 'noise'}

\section{Deterministic SARS model}

We suppose that the SARS outbreak in GTA has the pre-quarantine Model I (compare with another Model II-intra-quarantine). ${ }^{1}$ This Model I consist of the following compartments:

1. Susceptible $\mathrm{S}$ (individuals not yet infected).
2. Exposed E (susceptible who have become infected and are not yet infectious);

3. Infective I (exposed individuals who have become infected and can spread the SARS corona virus);

4. Hospitalized $U$ (infective who are in the immediate environment of HCWP (health care workers and patients); these individuals are not considered to pose any risk to the general public, but may infect HCWP).

5. Removed R (individuals who have been either exposed or infective, and who are considered to no longer be susceptible).

Thus, the Model I consists of 8 coupled nonlinear differential equations describing the transfer of individuals from one compartment to another.

The deterministic SARS Model I have the following look:

$$
\left\{\begin{array}{c}
\frac{d S_{g}(t)}{d t}=-a_{g} S_{g}(t)\left(I_{g}(t)+I_{h}(t)\right) \\
\frac{d S_{h}(t)}{d t}=-a_{h} S_{h}(t)\left(I_{g}(t)+I_{h}(t)\right)-a_{u} S_{h}(t)\left(U_{h}(\mathrm{t})+U_{g}(t)\right) \\
\frac{d E_{g}(t)}{d t}=a_{g} S_{g}(t)\left(I_{g}(t)+I_{h}(t)\right)-b_{g} E_{g}(t) \\
\frac{d E_{h}(t)}{d t}=-a_{h} S_{h}(t)\left(I_{g}(t)+I_{h}(t)\right)+a_{u} S_{h}(t)\left(U_{h}(t)+U_{g}(t)\right)-b_{h} E_{h}(t) \\
\frac{d I_{g}(t)}{d t}=b_{g} E_{g}(t)-c_{g} I_{g}(t)-r_{g} I_{g}(t) \\
\frac{d U_{g}(t)}{d t}=r_{g} I_{g}(t)-e_{g} U_{g}(t) \\
\frac{d I_{h}(t)}{d t}=b_{h} E_{h}(t)-c_{h} I_{h}(t)-r_{h} I_{h}(t) \\
\frac{d U_{h}(t)}{d t}=r_{h} I_{h}(t)-e_{h} U_{h}(t) \\
S_{g}(0)=S_{g}, S_{h}(0)=S_{h}, E_{g}(0)=E_{g}, E_{h}(0)=E_{h} \\
I_{g}(0)=I_{g}, I(0)=I_{h}, U_{g}(0)=U_{g}, U_{h}(0)=U_{h}
\end{array}\right.
$$


Here: a_g, a_h, a_u, are the transmission coefficients for the general public and HCWP infectives, and of hospitalized infective for HCWP, respectively; $b \_g$ and $b \_h$ are the transmission coefficients of exposed individuals to the infective class; $\mathrm{c} \_\mathrm{g}$ and $\mathrm{c} \_\mathrm{h}$ are the transmission coefficients of infective individuals to the removed class; $r$ g and $\mathrm{r} \_\mathrm{h}$ are the transmission coefficients of infectives to hospitalization; e_g and e_h are the transmission coefficients to the removed class, reflecting the effectiveness of treatments. The second equation in Model I describes the additional risk of HCWP resulting from their direct contact with SARS patients in the health-care setting. ${ }^{1}$

\section{Stochastic SARS model}

The stochastic SARS Model I in semi-Markov random media has the following look:

$$
\left\{\begin{array}{l}
\frac{d S_{g}(t)}{d t}=-a_{g}(y(t)) S_{g}\left(I_{g}(t)+I_{h}(t)\right) \\
\frac{d S_{h}(t)}{d t}=-a_{h}(y(t)) S_{h}(t)\left(I_{g}(t)+I_{h}(t)\right)-a_{u}(y(t)) S_{h}(t)\left(U_{h}(\mathrm{t})+U_{g}(t)\right) \\
\frac{d E_{g}(t)}{d t}=a_{g}(y(t)) S_{g}(t)\left(I_{g}(t)+I_{h}(t)\right)-b_{g}(y(t)) E_{g}(t) \\
\frac{d E_{h}(t)}{d t}=-a_{h}(y(t)) S_{h}(t)\left(I_{g}(t)+I_{h}(t)\right)+a_{u}(y(t)) S_{h}(t)\left(U_{h}(\mathrm{t})+U_{g}(t)\right)-b_{h}(y(t)) E_{h}(t) \\
\frac{d I_{g}(t)}{d t}=b_{g}(y(t)) E_{g}(t)-c_{g}(y(t)) I_{g}(t)-r_{g}(y(t)) I_{g}(t) \\
\frac{d I_{h}(t)}{d t}=b_{h}(y(t)) E_{h}(t)-c_{h}(y(t)) I_{h}(t)-r_{h}(y(t)) I_{h}(t) \\
\frac{d U_{g}(t)}{d t}=r_{g}(y(t)) I_{g}(t)-e_{g}(y(t)) U_{g}(t) \\
\frac{d U_{h}(t)}{d t}=r_{h}(y(t)) I_{h}(t)-e_{h}(y(t)) U_{h}(t) \\
S_{g}(0)=S_{g}, S_{h}(0)=S_{h}, E_{g}(0)=E_{g}, E_{h}(0)=E_{h} \\
I_{g}(0)=I_{g}, I_{h}(0)=I_{h}, U_{g}(0)=U_{g}, U_{h}(0)=U_{h}
\end{array}\right.
$$

where functions $\mathrm{a}_{\mathrm{g}}(\mathrm{y}) ; \mathrm{a}_{\mathrm{h}}(\mathrm{y}) ; \mathrm{a}_{\mathrm{u}}(\mathrm{y}) ; \mathrm{b}_{\mathrm{g}}(\mathrm{y}) ; \mathrm{b}_{\mathrm{h}}(\mathrm{y}) ; \mathrm{c}_{\mathrm{g}}(\mathrm{y}) ; \mathrm{c}_{\mathrm{h}}(\mathrm{y}) ; \mathrm{r}_{\mathrm{g}}(\mathrm{y}) ;$ $r_{h}(y) ; e_{g}(y) ; e_{h}(y)$ are continuous and bounded on Y: Therefore, we suppose that our coefficients are random, not constants, and, in general, are functions of some random parameter, in our case, semiMarkov random process.

\section{Stochastic SARS Model with Noise}

The stochastic SARS Model II with 'noise' has the following look:

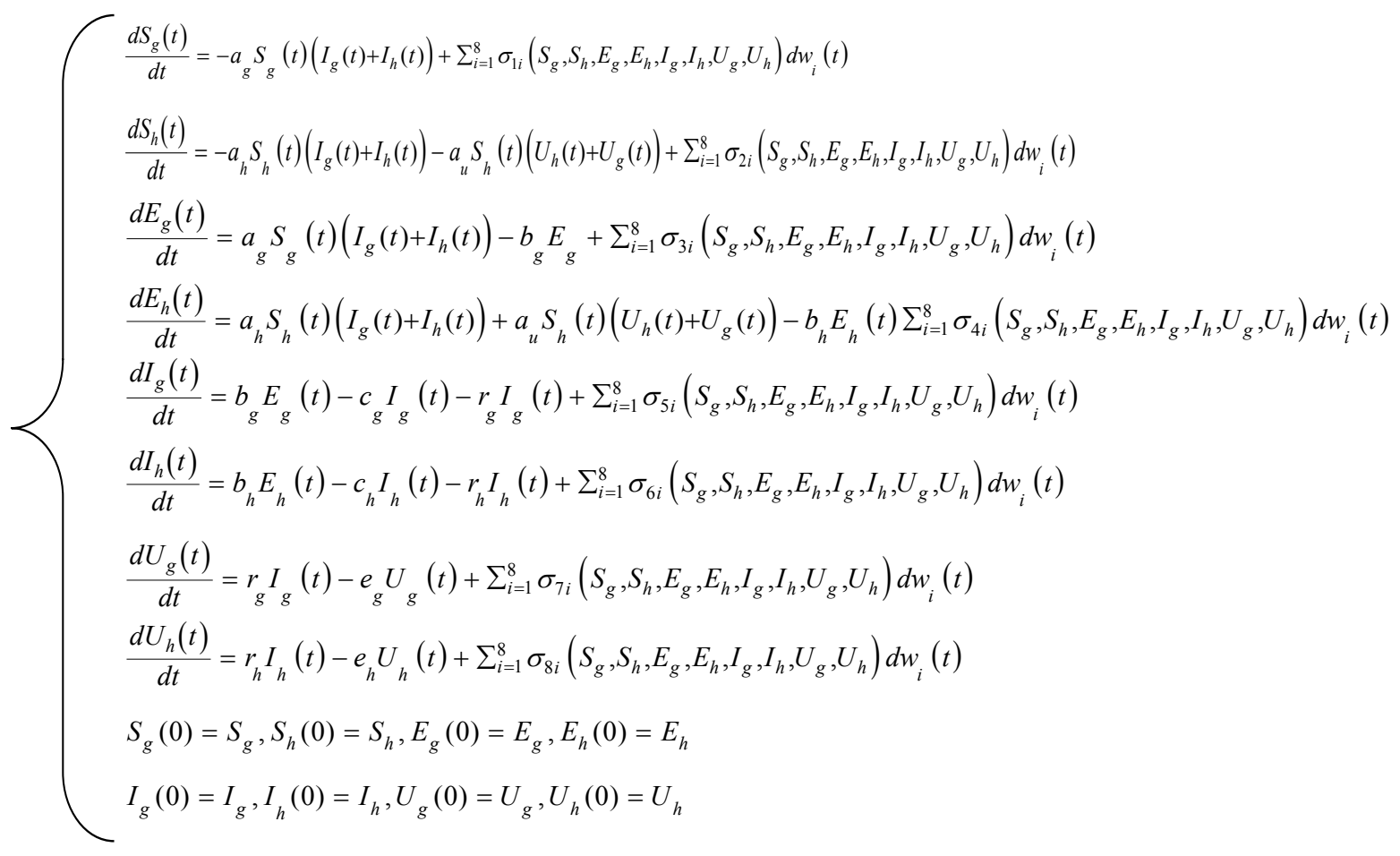


Here: $\mathrm{w}_{\mathrm{i}}(\mathrm{t}), \mathrm{i}=1 ; 2 ; 3 ;:: ; 8$; are independent Wiener processes, $\sigma_{i j}\left(S_{g} ; S_{h} ; E_{g} ; E_{h} ; I_{g} ; I_{h} ; U_{g} ; U_{h}\right) ; i, j=1,2, \ldots, 8$; are entries of diffusion matrix.

\section{Acknowledgement}

None.

\section{Conflict of interest}

None.

\section{References}

1. Nasel I. The threshold concept in stochastic epidemic and endemic models. In Epidemic Models. 1985.

2. Nasel I. Stochastic models of some endemic infections. Math Biosci. 2002;179:1-19

3. Ball F, Lyne O. Stochastic multi-type SIR epidemics among a population partitioned into households. Advances in Applied Probability. 2001;33:99-123.
4. Hethcote HW. The mathematics of infectious diseases. SIAM re-view. 2000;42:599-653.

5. Allen L, Burguin A. Comparison of deterministic and stochastic SIS and SIR models in discrete time. Math Biosci. 2000;163: 1-33.

6. Isham V, Medley G. Models for Infectious Human diseases: their Structure and Relation to Data. Cambridge: Cambridge University Press; 1996.

7. Swishchuk A, Wu J. Evolution of Biological Systems in Random Media: Limit Theorems and Stability. Kluver Academic Publishers; 2003.

8. Swishchuk A, Limnios N, Svishchuk M. Stochastic SARS model: Approximations and Stability. European J of Epidemiology. 2015.

9. Svishchuk M. Stochastic endemic model in random media. 2015.

10. Swishchuk A, Svishchuk M, Limnios N. Stochastic stability of vector SDEs with applications to a stochastic epidemic models. $J$ of Mathematics. 2015 . 